\title{
PAPYROLOGICA FLORENTINA
}

a cura di Rosario Pintaudi

Volume VII

\section{MISCELLANEA PAPYROLOGICA}

\section{Estratto}

EDIZIONI GONNELLI

Firenze 1980 
Proprietà letteraria riservata.

Tipolitografia Latini Firenze - Finito di stampare nel luglio 1980. 


\section{PAPYRUS DOCUMENTATION IN EGYPT FROM} CONSTANTINE TO JUSTINIAN

In a remarkable and penetrating paper at the Eleventh International Congress of Papyrology, Roger Rémondon described and analyzed the distribution of dated papyri from the fifth century and, for the sake of context, from the forty years on either side of it, thus $360-540$ in all (1). From it he created graphs showing the distribution, and on the basis of these he formulated a theory explaining the enormous decline in the fifth century and significant revival in the sixth by means of the evolution of institutions: a reduction of administration and official activity to the profit of large private landowners.

It seems to us of interest to examine the problem once again to see if another decade and a half, in which notably considerable numbers of Byzantine papyri from Berlin, Strasbourg and Vienna have been published, have affected the picture. The lists compiled in our Chronological Systems of Byzantine Egypt and Regnal Formulas in Byzantine Egypt provide, when combined, a list of all texts dated by regnal years, consulate, Oxyrhynchite era, or a combination of these, for the period 284-641. In the present article we take up the period treated by Rémondon-slightly enlarged by the period 337-360 (2).

The graph below presents our results, for the whole of Egypt and then for the Arsinoite, Herakleopolite, Hermopolite and Oxyrhynchite nomes individually, in the way that Rémondon did. In each case we show the figures given by Rémondon by the broken line, our own with a solid one. The reader will notice at once [1] that the lines are very similar in shape, and [2] that Rémondon's is sometimes higher than ours.

The first of these facts is not particularly a surprise for anyone who looks at Rémondon's Graph 1, where the lines for 1965 and 1925 are given.

(1) L'Egypte au 5e siècle de notre ère: les sources papyrologiques et leurs problèmes, Atti dell'XI. Congr. [1965] di Papirologia (Milan 1966) 135-48 plus one plate of graphs.

(2) We have treated the period 541-641 in Bulletin of the Egyptological Seminar [New York] 1 (1979) 5-10. A discussion of 284-337 will appear on another occasion. 
That for 1965 is almost a perfect duplicate of that for 1925, lifted several notches. The essential similarity of our graph to Rémondon's 1965 graph comes to confirm that the basic shape of the graph is a real and permanent fact of papyrological documentation, and not only an accident of discovery or publication. Such a conclusion fully supports the thesis presented by Rémondon concerning the meaning of the decline of numbers in the fifth century and recovery in the sixth.

The other fact, however, may astonish and disconcert: has the number of published documents for any decade declined in the last 15 years? It is of course a priori possible: a misdated document now relocated will leave a gap in the year to which it was wrongly assigned, and the critical work of the last few years has corrected a number of dates (3). But there is more involved than that. We must, therefore, examine how Rémondon's charts were constructed. Rémondon tells us (pp. 135-36) that he excluded texts not ' exactly' dated and that he excluded ostraka and inscriptions. Now the latter exclusions are insignificant: there are only two dated ostraka from this period to exclude, and scarcely any inscriptions. The question then is what is meant by 'exactement datés'. In our own lists we have taken it to mean dated to a particular year or to a meaningfully narrow range of years (e.g. P.Laur. II 27: between 487 and 491). That Rémondon meant something much less precise is indicated by his statement "Par exemple, nous n'avons aucun papyrus daté d'Antaioupolis entre 380 et le début du 6e siècle ", glossed with the note " BGU 974, et les pièces les plus anciennes des archives de la famille de Dioscore d'Aphrodito (P. Maspero 67135, 67327; PSI 935; P.Lond. 1702 ; P.Flor. 281)".

Now this choice is peculiar: P.Cair.Masp. I 67001, 67100 and 67101, P.Flor. III 279 and 280 would have been better choices than P.Flor. III 281, which is later than any of them. P.Cair.Masp. II 67135 has as dating criterion only the second indiction (coming); 67327 is dated to Mesore of a third indiction; PSI 935 is a receipt for the second indiction; P.Lond. 1702 refers to rent for the sixth indiction. None of these has a consular date. Any exact date depends on prosopographical and archival study. Now such investigation is valuable and the dates arrived at may be fairly secure: but the use of such texts for a list of this kind introduces an element of subjectivity and-what is worse-makes it impossible to verify the graph presented, for Rémondon gives no list of what documents he includes nor any references or argument for those the date of which rests on inference. We are thus unable to attempt any detailed reconciliation of our figures with Rémondon's.

(3) A bibliography of our efforts in the area of Byzantine chronology is given in GRBS 20 (1979) 279-95, especially 279 n. 1. 
Another factor, regrettably, may be at work. Rémondon says (p. 136), "Antinoé n'a livré pour le 5e siècle que deux papyrus isolés, qui sont de 430 ". His references are to PSI 1239 and SB 7996. But these are the same papyrus in two publications (4). Again, the absence of any list makes further verification impossible. Rémondon cites also P.Sorbonne inv. 2253 (143 n. 5, 145 n. 3) of A.D. 502 and presumably included it in his figures, though it is still unpublished (5). We cannot say if there were other such inedita in the figures. It will by now be clear why we have regarded the inclusion of our own lists (Appendix) as anything but a waste of space.

An examination of the graphs by nomes shows that while some peaks and valleys have become flatter and others sharper and deeper, little has changed in the overall shape of the curves. There is one significant exception, the Hermopolite Nome between 460 and 520 , where comparatively large archival masses in BGU and P.Stras. have come to augment the totals and provide a rarity in this period, archival material (6). Rémondon had commented (p. 144) on the virtual disappearance of archives in the fifth-century papyri. This absence is somewhat less striking now, but the situation is far from being like the 4th or 6th century. Whatever future finds may do to enlarge the documentation of the fifth century, they do not seem likely to lead it to overshadow the fourth and sixth centuries, for which recent publications are more numerous still.

(4) This oversight was already noticed, as we now see, by D. Bonneau in Hommages S. Sauneron (Cairo 1979) 4-5 n. 7.

(5) We will publish this papyrus elsewhere through the kind permission of Professor Jean Scherer.

(6) Cf. especially P.Stras. $470-480$ (Church of the Resurrection) and BGU XII (archive of Taurinos): see esp. pp. XIX-XXIV.

\section{APPENDIX}

\section{LIST OF DOCUMENTS INCLUDED IN TABULATION}

Introductory note: No system of counting documents is quite satisfactory, but we must state a few of our principles. Texts in which multiple documents are recorded are counted once for each year in which there is a date given in it. Thus a text with receipts in 316 , two in 317 , five in 318 , ten in 319 , and one in 320 is counted once for each year. Retrospective 
references to years (" In the consulate of $\mathrm{X}$ and $\mathrm{Y}$ the defendant attacked my grandmother ") are not counted.

It is obvious that many documents dated inferentially have a strong probability or near-certainty for exact dating. These are, however, not included. Noteworthy are many tax receipts from Karanis and much of the Abinnaeus archive. In all likelihood, the net result of any such documents if they were included would be to inflate the periods with an already high number of texts and thus exaggerate the curves. The reader should keep this in mind.

Finally, for provenance we have used the place where the document was written, so far as that can be determined. Many documents were found at locations different from that of writing, but we have not taken these as the provenance. This practice is again arguable, but a decision had to be made and we consider the ancient place of writing the more important.

References to $C N B D$ refer to our "Chronological Notes on Byzantine Documents " in BASP 15 (1978) and following volumes.

An asterisk marking a reference indicates a papyrus not listed in $C S B E$, app. D.

\section{Antaiopolite}

380: BGU III 974. 506: P. Cair.Masp. I 67100. 511: P.Cair.Masp. I 67101. 514: P.Flor. III 279, P.Cair.Masp. I 67001, P.Flor. III 280. 515: P.Cair.Masp. III 67306. 517: P.Flor. III 281. 520: P.Lond. V 1699, P.Flor. III 282. 521: P.Cair. Masp. III 67328. 522: P.Ross.Georg. III 33. 523: P.Lond. V 1687, 1688. $\quad 524: P$. Cair.Masp. I 67117, P.Flor. III 342, PSI VIII 931*. $\quad$ 525: P.Cair.Masp. I 67107 (or 540 ?), II 67125, II 67254. 526: P.Michael. 43, P.Cair.Masp. I 67102, 67103. 527: P.Michael. 44, P.Cair.Masp. III 67300, P.Lond. V 1689, 1690, P.Mich. XIII 670. 528: P.Cair.Masp. I 67091. 530: P.Cair.Masp. I 67104, III 67301. 532: P.Lond. V 1691, P.Cair.Masp. I 67105. 535: P.Cair.Masp. III 67296, 67297. 536: P.Lond. V 1841, P.Flor. III 283. 537: P.Cair.Masp. I 67123, P.Ross.Georg. III 36. $\quad$ 538: P. Cair.Masp. II 67252, P. Flor. III 284, SB III 6266 =6704, P.Cair.Masp. I 67106, PSI VIII 933*. 540: P.Michael. 45.

Antinoopolite

347: P.Ant. I 31. 364: P.Ryl. IV 662. 375: P.Lips. 61. 388: P.Lips. 63. 
390: P.Ant. II 102. 430: PSI XII 1239. 454: SPP I, p. 7 (II). 529-530: P.Stras. 317. 531: $S B \times 10524 . \quad 537: S B$ V 8029.

\section{Arsinoite}

338: P.Sakaon 70. 339: P.Col. VII 175, BGU XIII 2296. 340: P.Col. VII 148, 149. 342: P.Sakaon 46, P.Abinn. 44, P.Coll.Youtie II 78, BGU IV 1049*. 343: $S B$ VI 9622, P.Abinn. 45, 46. 345: P.Abinn. 58, 59. 346: P.Abinn. 47, 48, 49, 50, 51, 52, 53, 54, 60, 61. 348: BGU II 405, 406, III 917. 349: P.Würzb. 16. 350: P.Abinn. 62, 63. 351: P.Abinn. 55, CPR V 12. 352: P.Stras. I 9. 358 (or 343 ?): P.Ross.Georg. III 28. 359: BGU III 909. 362: P.Flor. I 30. 363: P. Stras. 131. 372: P.Col. VII 182, 183, 184. 373: P.Mert. I 37, P.NYU I 24. 374: P.Gen. 66, $B G U$ XII 2332, $S B$ VI 9311 (or 375; ef. $C N B D$ VII 71). 377: ZPE 24 (1977) 140. 378: P.Grent. I 54. 382/383: P.Gen. 67. 382: P.Gen. 68. 383: P.Gen. 12. 386: P.Gen. 69. 391: P.Stras. 142. 392: P.Gron. 9. 398: P.Flor. I 66. $400: S B$ VI 9359 (cf. $C N B D$ IV 52). 407: $S B$ I 5159. 409/410: $S P P$ $\mathrm{XX}$ 115. 415. SPP XX 90. 417: P.Got. 39. 439: P.Haun. inv. 318. 441/ 442: BGU II 609. 454: P.Würzb. 17. 465: SB I 4821 (cf. $C N B D$ IV 50). 486: $S B$ I 4481. 487: SPP XX 128, P.Amh. II 148. 487-491: P.Laur. II 27. 493: P.Grent. I 55. 498: P.Lond. I 113, 5(a), p. 210. 503: P.Ross.Georg. V 31. 504: P. Ross.Georg. III 32: 511: SPP XX 135. 512: $S B$ I 5174. 518: SPP XX 131 . 530: $B G U$ II 369. 531: SPP XX 139. 533: $S P P \mathrm{XX} \mathrm{140,} S B$ I 4663 . 536: $P$. Grent. II 85.

\section{Herakleopolite}

347: P.Athen. 34. 350: P.Oxf. 6. 363: BGU III 939. 372: P.Vindob.Sijp. 13. 376: $B G U$ III 941. 381: SPP XX 103. 384/5: BGU III 938. 389: $B G U$ III 943. 398: BGU III 940. 411: SPP XX 117. 414/417/420: P.Vindob.Sijp. 9. 415: P.Mich. XI 613. 421: Pap.Lugd.Bat. XIII 13. 435: Pap.Lugd.Bat. XIII 15. 445: SPP XX 123. 463: P.Vindob.Sijp. 7 (CNBD III 32). 463: SPP XX 127. 475: $C P R$ V 14. 478: $C P R$ V 15 (cf. P.Köln III 152 introd.). 484: PSI III 183. 492: $S B$ VI 9152. 497: $S P P \mathrm{XX}$ 129. 522: $S P P \mathrm{XX} 137 . \quad 534: S B$ VII 19876. 538: P.Michael. 126 (ostr.). 540: SB VIII 9773. 
Hermopolite

337: SPP XX 88. 338: P.Vindob.Sijp. 1. 339: CPR V 9. 340: P.Vindob. Sijp. 4, BGU I 21, P.Cair.Goodsp. 12, P.Vindob.Sijp. 5, CPR VII 16*. 341: P.Cair. Goodsp. 13, SPP XX 89, P.Flor. I 17. 342: P.Flor. I 34 (?). $\quad 343:$ SPP II, p. 34, P.Cair.Goodsp. 14. 343/344: ZPE 20 (1976) 158. 345: P.Lond. III 1246 (p. 224), P.Lond. III 1247 (p. 225), P.Lond. III 1248 (p. 226). 346 : P.Lond. III 1249 (p. 227), P.Herm. 21 and 54, P.Lond. III 979 (p. 234), P.Oslo III 113. 347: P.Cair.Preis. 39. 348: SPP XX 98. 349: P.Amh. II 140. 350: P.Amh. II 139, 141*. 357: P.Lond. III 1245 (p. 228). 362: Cd'E 49 (1974) 342, P.Cair.Preis. 2 and 3, P.Cair.Goodsp. 15. 363: P.Lond. V 1651. 366: P.Flor. I 84. 368: P.Lond. III 1113 (p. lvii), P.Lips. 33 II. $\quad 369$ : P.Stras. 272. 370: P.Flor. I 43. 372: P.Lips. 85, BGU IV 1092. 373: P.Lips. 86, P.Lond. V 1648, 1649, 1822, P.F'lor. III 320, P.Lond. V 1650, 1828, P.Lips. 34 verso. $\quad 374$ : P.Lips. 23 (cf. CNBD IV 38). 375: BGU XII 2148 (cf. CNBD I 1). 375-377: P.Flor. I 95. 376: P.Flor. I 52. $\quad 377$ : P.Lips. 17. 379: P.Lips. 13. 380: P.Flor. I 75, CPR VII 19*. 381: P.Lips. 20, 28. 382: P. Lond. III 980 descr. (p. 1), P.Lips. 21. 384-385: P.Lips. $62 . \quad 388$ : SB VIII 9907. P.Lips. 22. 389: P.Lips. 37, P.Ross.Georg. IIJ 30. 389-390: P.Lips. $38 . \quad 390$ : W.Chr. 434, P.Lips. 39, 65. 391: P.Lips. 14, 42 (cf. CNBD IV 39), P.Ross.Georg. V 60R (p. 176). 392: P.Herm. 19. 394: P.Herm. 22. 397: PSI I 34, P.Giss. 52. 398: P.Lips. 56, P.Herm. 52, 53. 399: P.Giss. 104. 402:P.Grenf. II 80. $\quad 403: P$. Grenf. II 81, 81a. 405: SB VIII 9931. 410: P.Herm. 69. 417: P.Berl.Zill. 5. 425: P.Stras. 639. 426: BGU XII 2137. 428: P.Flor. III 314. 429: PSI JII 245. 430: $B G U$ XII 2138. 432: BGU XI 2139, 2140. 435: P.Stras. I 1 (CNBD VI 63), P.Flor. III 315. 439: SPP XX 121, $C P R$ VI 6. 446: $B G U$ XII 2141. 448: $P$. Flor. III 311 (see ZPE 36 [1979] 105-106). 449: P.Flor. III 313. 453: P.Vindob. Sijp. 11. 454: P.Lond. V 1773. 457: BGU XII 2146. 462 ?: M.Chr. 71. 464: BGU XII 2147. 465: PSI VII 768. 470: BGU XII 2149, P.Herm. 61. 471:P.Bad. IV 91b. 472: BGU XII 2150, P.Lond. V 1793, P.Stras. 148. 476: BGU XII 2151. 477: SB III 7167 (CNBD VI 63). 480: PSI VI 703. 481: BGU XII 2155. 483:P. Lond. V 1896, BGU XIJ 2156. 485: P.Coll.Youtie II 89, BGU XII 2157, BGU XII 2159. 486: $C P R$ V 16. 488: P.Lond. V 1794 (cf. $C N B D$ IV 34), BGU XII 2160. 491: $B G U$ XII 2162*, P.Flor. I 94. 492: CPR VII 40*. 494: $B G U$ XII 2164. 496: SB VIII 9776. 497: SB V 7758*, P.Stras. 470 (?). 498: BGU XII 2173, Talanta 6 
(1975) 50, P.Lond. III 1303 descr. (p. lxxii). 500: P.Herm. 79, P.Stras. $471 . \quad 501$ : P.Amst. I 45, BGU XII 2174. 504: BGU XII 2180, Pap.Lugd.Bat. XVII $17 . \quad 505$ : P.Stras. 471 bis, 578. 506: P.Lond. III 867 descr. (p. lxi), P.Stras. 656*. 507: P. Lond. 1313 (p. 256). 509: BGU XII 2181, P.Vindob. Sal. 9. 510: P.Berl.Frisk 5, BGU XII 2182. 512: BGU XII 2185, SB IV 7369, P.Stras. 483. 513: P.Coll. Youtie II 90, CPR VII 43*, Festschr.Berlin Museum 409. 514: BGU XII 2186. 515: SPP XX 126. 517: P.Lond. III 994 (p. 259). 520: BGU XII 2187, PSI IV 296. 521: P.Stras. 579. 525: P.Flor. III 323, BGU IV 1094. 526: BGU XII 2188. 533/534: P.Stras. 472 (cf. CNBD VI 63). 537: P.Amst. I 47, P.Grent. I 56, P.Stras. 473. 538: P.Lond. III $998+999,1000$, P.Stras. $481 . \quad 539:$ P.Lond. III 1001. 540: PSI III 188.

\section{Oxyrhynchite}

337: P.Oxy. XLV 3266. 338: P.Oxy. I 67, 85, 86, VI 892, XII 1575, XXXI 2571. 339: PSI III 215. 340-341: PSI VII 782. 341: P.Oxy. VI 991, PSI VII 781, P.Oxy. XII 1559. 342: P.Oxy. I 87, P.Harr. 65, P.Oxy. XIV 1627. 344:P. Princ. II $81=$ III 181. 345: P.Wisc. I 12, P.Genova I 22, P.Harr. 82. 345-346: PSI VIII 948. 346: ZPE 23 (1977) 139, P.Oxy. VI 897. 346-347: Pap.Lugd.Bat. XIX 16, P.Mert. I 34. 347: P.Oxy. IX 1190, XIV 1751, XLIII 3146. 348: P.Mert. I 35. 350: P.Oxy. XIX 2233. 351: PSI VI 707, P.Oxy. XII 1431. 353: P.Oxy. XIV 1632, P.Coll.Youtie II 83. 354/355: PSI IX 1077 (cf. RFBE 76). 356: PSI IX 1078. 357: P.Oxy. I 66. 360: P.Oxy. VII 1056, VIII 1103, XIV 1695, XX 2267, PSI V 467, VI 711, P.Mert. I 36. 362: P.Oxy. I 93, VII 1057. 363: P.Oxy. VIII 1116. 363/364: P.Harr. 145. 364: PSI I 90, P.Lond. V 1655. 365: SB III 6612. 370: P.Oxy. XVII 2110. 373: P.Oxy. XLVI 3308*, 3309*. 374: P.Oxy. XLVI 3310. 377: PSI IV 287, SymbOsl 52 (1977) 107 (or later). $378: B G U$ XIII 2339. 379: P.Oxy. XIV 1752. 380: PSI VII 813. 381: P.Oxy. VII 1041, PSI X 1108. 382: SB IV 7445. 386: P.Oxy. XXXIV 2715. 388/393: SB VIII 9825. 389: PSI IX 1073. 390: P.Oxy. XIV 1753, PSI VIII 884. 391: PSI XV 1566. 392: PSI VI 698, P.Oxy. VII 1033. 393: P.Rein. II 92. 394: P.Oxy. XIV 1712. 396: P. Oxy. VIII 1133, P.Flor. I 39. 399: P.Oxy. X 1329. 400: P.Oxy. XLIV 3203, PSI IX 1074. 403: P.Oxy. X 1319. 406. CPR VII 39, P.Mert. I 41. 407: P.Oxy. VIII 1122, P.Harr. 90. 410: P.Wisc. II 63. 412: P.Mich. XI 611. 413: P.Oxy. VI 992, X 1322. 
416 or 417 ?: P.Oxy. X 1334. 418: P.Köln II 102. 419: PSI XIII 1365, P.Oxy. XVI 1953. 420: P.Oxy. VIII 1136, XVI 1973, PSI XIII 1340. 421: P.Oxy. VIII 1134, Pap.Lugd.Bat: XIII 8 (cf. CNBD I 3). 423: PSI I 87, VI 689. 426: BGU III 936, $P$. Oslo II 35. 427: P.Oxy. XVI 1880, 1881, 1967. 430: P.Oxy. XVI 1957. 432: P. Rein. II 105. 433: SB XII 11181. 434: P.Lond. V 1777, P.Oxy. XVI 1879. 436: PSI VI 708. 438: P.Köln II 103. 440: P.Harr. 87. 441: P.Mil. I 64. 443: P. Oxy. VI 913. 444: P.Oxy. VII 1037, P.Harr. 149. 449: P.Oxy. VIII 1129, P.Mil. I 45. 453: P.Harr. 78. 454: Pap.Lugd.Bat. XIII 1, PSI X 1114. 455: P.Gron. Amst. 1. 456: P.Yale I 71. 458: P.Oxy. XXXIV 2718, PSI IX 1075. 461: P. Oxy. XVI 1878. 462: PSI III 175. 466: P.Oxy. XVI 2001. 468: P.Wisc. I 10. 469: P.Oxy. XXXIV 2724. 470: P.Lond. V 1798. 475: P.Oxy. XVI 1899. 475/ 476: StudPap 13 (1974) 81. 476: P.Oxy. XVI 1958. 480: P.Wisc. II 64. 482: P.Oxy. X 1335. 484: P.Oxy. VIII 1130, XVI 1969, P.Harr. 91. 486: P.Oxy. VI 914. 487: P.Oxy. XVI 1950, 1961. 488: P.Oxy. XVI 1888. 489: Aegyptus 56 (1976) 27, P.Flor. III 325. 492: ZPE 30 (1978) 205*. 495: P.Oxy. XVI 1891. 496: P.Oxy. XVI 1889, 1975. 497: P.Oxy. X 1320, XVI 1982. 498: P.Oxy. XIX 2237. 499: P.Oxy. XVI 1959. 500: P.Oxy. XVI 1962. 499 or 500: P.Oxy. VI 994. 503: P.Oxy. I 141. 504: P.Oxy. XVI 1883, 1884. 505: P.Oxy. XVI 1966, 1994. 508: P.Oxy. XVI 1890. 509: P.Oxy. XVI 1885. 511: P.Oxy. XVI 1960. 513: P.Erl. 78, P.Oxy. XVI 2005. 514: P.Mich. XI 612. 516: P.Lond. V 1797 (cf. CNBD III 35). 518: PSI V 466, P.Oxy. XVI 1964. 520: P.Mert. III 124. 522-523: Pap.Lugd.Bat. XVII 10. 523: P.Oxy. XVI 1984. 524: SB V 8264, P.Oxy. XVI 1946. 525: P.Iand. III 43. 526: PSI III 246. 528: P.Oxy. XVI 1900. 530: P.Oxy. XXXVI 2779. 534: PSI III 216, P.Oxy. I 142. 535: P.Oxy. I 143, XVI 1893, 1983, P.Erl. 75, P.Lond. III 780 (p. 245). 534/535: P.Oxy. I 206. 538: P.Oxy. XVI 1887, 1974.

Other provenances

Hypselite: 371: P.Lips. 58. 372: P.Lips. 48, 49, 51.

Panopolite: 337: P.Panop. 12. 339: P.Panop. 9, $13 . \quad 341:$ P.Panop. $10 . \quad 338$ 345: P.Panop. 19. 348: Ill.Class.Stud. 2 (1977) 185. 371: P.Lips. 45, 46, 59. 372: P.Lips. 47. $\quad$ 502: P.Stras. 229. 
Diospolite (Parva): 339: P.Ant. I 32.

Apollinopolite (Parva): 455: SPP I, p. 8 (iii).

Cynopolite: 464: P.Oxy. VI 902.

Alexandria: 344: P.Abinn. 2. 397: P.Oxy. XXIV 2408. 421: SPP XX 114.

Elephantine: 355: P.Oxy. IV, p. 202 (cf. CNBD I 2).

Great Oasis: 376/378: P.Lips. 36.

Hermonthis: 338: P.Lips. 97. 503/504: P.Herm. 28. 525: BGU II 673.

Nilopolis: 343 : P.Mil. 66.

Lycopolis: 481 : P.Princ. II $82 . \quad 519$ ?: P.Stras. III' 133.

Ptolemais: 372: P.Lips. 50.

Syene: 493: P.Lond. V 1855.

Outside Egypt: 359: BGU I 316 (Askalon). 510: SB I 5941 (Caesarea ?).

\section{Provenance unknown}

338: P.Lond. III 651 descr., P.Ryl. IV 660. 339: P.Lond. III 1257 descr. 341 : P.Würzb. 15. 345: P.Princ. III 183. 346: WO II 1309. 354:P.Stras. 329. 357: SPP XX 101 (cf. CNBD I 10). 360 ?: P.Ross.Georg. V 29. 365-373: Aegyptus 56 (1976) 60. 367: ZPE 23 (1977) 218 (cf. CNBD IV 42). 371: P.Stras. 243. 372: P.Lips. 52, 53. 373: Aegyptus 54 (1974) 112. 374/375: SPP XX 102. 376: P. Stras. 596. 383: SPP XX 104. 387: Aegyptus 54 (1974) 83. 395/396: CPR V 13. 396: CPLat. 230. 397: SB XII 10932 (CNBD VI 63). 399: CPLat. 199. 397/ 403: P.Stras. 255. 401: $S B$ VIII 9774, SPP XX 113. 422: SPP XX 118. 424: $S B$ XII 11023. 428: P.Vindob.Tandem. 6. 444: P.Harr. 86. $\quad 454: S B$ X 10523. 481: P.Lond. III 991 (p. 258) (ef. CNBD IV 40). 505: SPP XX $130 . \quad$ 507: P.Lond. III 992 (p. 253). 508/509 ?: $C P R$ VI 8. 509: P.Lond. III 1307 deser. 514: P. Lond. III 993 descr. 525: P.Lond. III 1306 descr. 535: P. Giss. I $121 . \quad 536$ : SPP XX 141. 539: P.Cair.Masp. II 67255. 


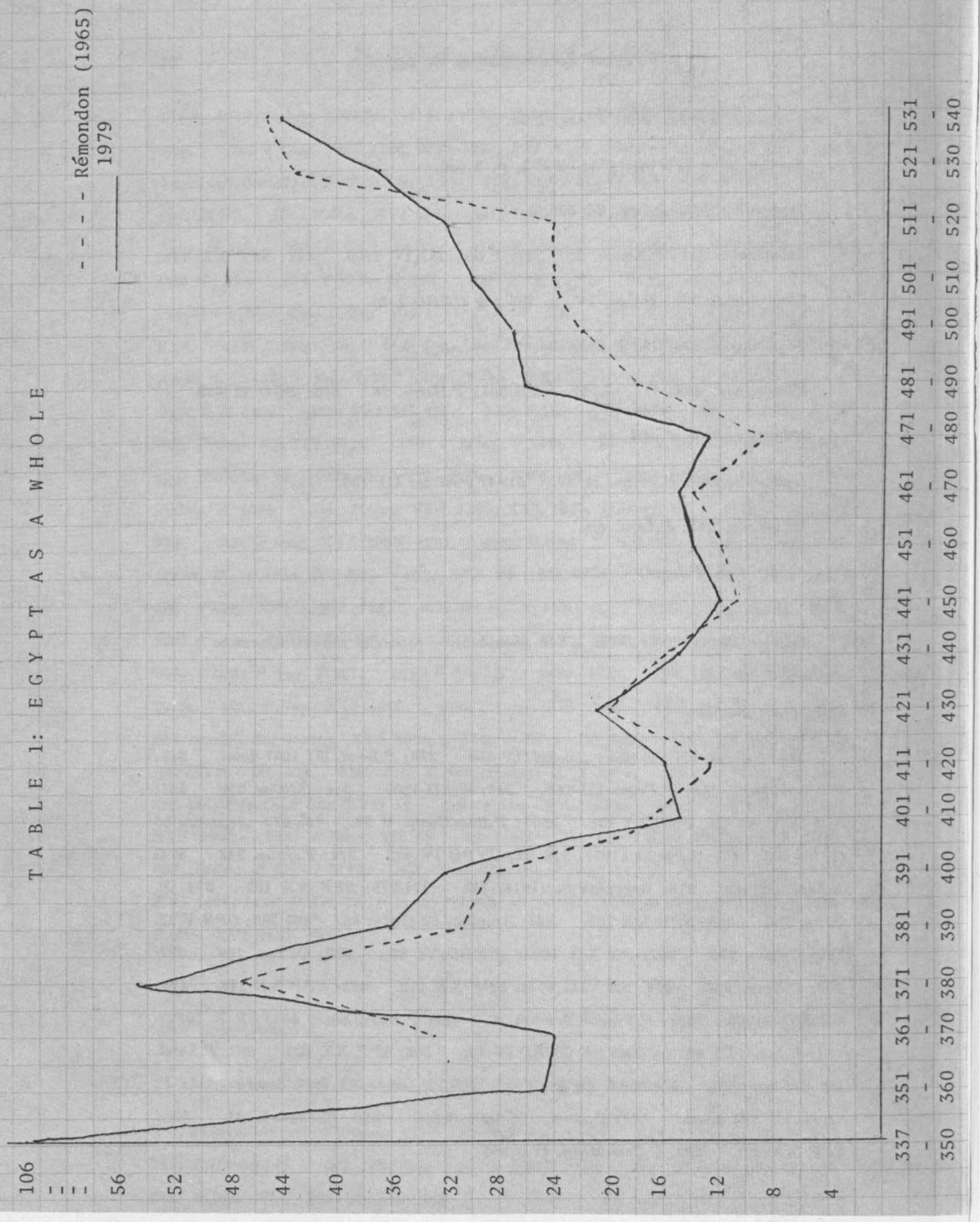




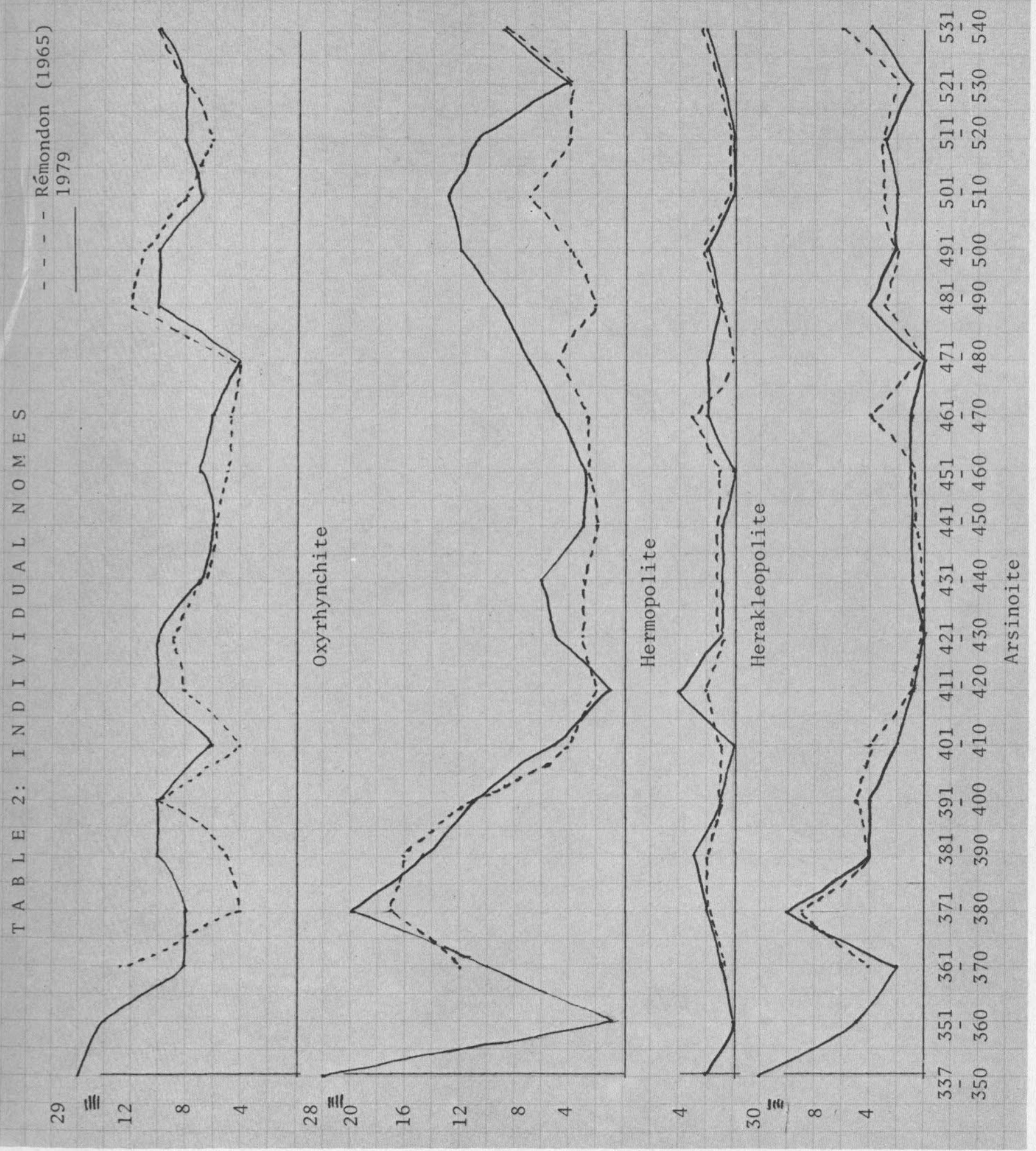

